Research Article

\title{
Wireless Sensor Networks Assist Intelligent Tourism Traffic Navigation and Tourist Positioning
}

\author{
Jin Sun \\ Henan Vocational College of Information and Statistics, Zhengzhou, Henan 450000, China \\ Correspondence should be addressed to Jin Sun; 20150367@stu.nmu.edu.cn
}

Received 17 September 2021; Accepted 5 October 2021; Published 26 October 2021

Academic Editor: Guolong Shi

Copyright (C) 2021 Jin Sun. This is an open access article distributed under the Creative Commons Attribution License, which permits unrestricted use, distribution, and reproduction in any medium, provided the original work is properly cited.

\begin{abstract}
With the booming development of tourism, more and more tourists visit scenic spots; followed by dense personnel in scenic spots, tourists' comfort is not high. This paper introduces the structure diagram, function structure diagram, and database design of an intelligent tourism navigation system based on a wireless sensor network in detail and explains each function module of an intelligent tourism system based on a wireless sensor network in detail. Through the wireless sensor network to obtain the positioning data of tourists in tourist attractions, this article realizes the intelligent traffic navigation in the intelligent tourism navigation system and finally realizes the diversion of tourists. An intelligent travel navigation system based on the wireless sensor network is implemented on Android. Finally, the intelligent travel navigation system based on the wireless sensor network is tested. The results show that the system can meet the real-time demand, the system positioning accuracy is high, and the system can effectively realize the flow analysis and public security warning.
\end{abstract}

\section{Introduction}

With the development of tourism, more and more tourists visit tourist attractions, which will lead to the phenomenon of crowded tourists and often stranded tourists, which will bring extremely bad experience to tourists and reduce their comfort [1]. In smart phones and other terminals, tourists often use navigation software such as Baidu Maps and AutoNavi for navigation. Although Baidu Maps, AutoNavi, and other navigation software can provide traffic data, they all have a prerequisite requirement that they must have a good network in the process of use. However, there may be some problems with network signals on the way of tourism. For example, the intensity of network information has a great relationship with the geographical location of tourist attractions. Network signals in tourist attractions in cities are often better than those in tourist attractions with high mountains. Like the Golden Map navigation software, the route of tourist attractions in Baidu Maps may not provide accurate detailed information. It is easy to get stuck in the tourist attractions of the query time, and the software cannot provide real-time traffic information and navigation informa- tion. This also has some problems for the real-time effectiveness of navigation within the scenic area. Therefore, it is necessary to provide an intelligent tourism navigation system with a good network for tourists to query and use in real time.

At present, the statistics of people flow mainly rely on base station positioning, and the low positioning accuracy leads to a large error in the statistics of people flow, which cannot well support more refined park management, such as reminding tourists of the waiting time in line and the safety situation and prediction of the park [2]. Compared with the traditional base station positioning and threepoint positioning technology, the real-time intelligent positioning system will use wireless sensor network technology to collect a large amount of longitude and latitude and signal strength information data in real time. The information base of signal intensity at different positions is constructed, and the end user will continuously report the time series measurement information in the process of use. The most matched information is selected from the information base, and weighted average, clustering, and other techniques are used to denoise the location results. Determining the 
geographical location of mobile terminal users can effectively improve positioning accuracy for more accurate geographic information services [3]. The establishment of a real-time positioning system will be used in fine-grained network optimization analysis, tourist flow analysis, and public security early warning, which is bound to have a wide range of application value and practical significance.

A collection of all independent sensor nodes in the area is called the wireless sensor network (WSN); because of the specialty of wireless sensor network application scenarios, basically these sensor nodes are low-power devices and nodes can communicate with each other, so that the whole network would have perception, computing, and communications capacity [4]. WSN has a wide range of applications, including environment monitoring, task monitoring, machinery control, medical monitoring, industrial monitoring, and tracking. Wireless sensor networks can not only make people perceive the world objectively but also explore the potential of mobile Internet and artificial intelligence. The communication modes of the wireless sensor network mainly include Zigbee, WiFi, and LoRa. Zigbee is a shortdistance and low-rate wireless communication technology. In the general frequency band of $2.4 \mathrm{GHz}, \mathrm{WiFi}$ and Bluetooth are also used, which are prone to interference. In addition, Zigbee has a high frequency, which degrades quickly in the process of wireless signal transmission, and has complex networking and poor penetration. The maximum likelihood estimation legal position algorithm has a great application in RSSI-based ranging location. Da Costa Liberato et al. compare the routing reference node to consider whether ranging error is considered or not, and the obtained results can better reflect the positioning effect [5]. Without considering other parameters in the positioning model, Gretzel and Koo calculate ranging values based on the principle of dimensionality reduction and then integrate the maximum likelihood estimation positioning algorithm and Taylor series to calculate the location of unknown nodes, but this method is relatively complicated [6]. Liao uses the maximum likelihood estimation algorithm to roughly estimate the coordinate positions of unknown nodes and then uses the weighted centroid localization algorithm to estimate and refine the coordinates of unknown nodes, so as to reduce the estimation error [7]. Liao and Nong adopt the extended Kalman filter method (EKF), but the initial state of the EKF filter is difficult to determine, and the calculation is complex and errorprone, resulting in unstable or even error filtering [8]. A wireless network sensor is an embedded device with limited resources. Compared with the traditional network and traditional sensor acquisition system, it has different characteristics and performance. As a new research technology, it presents a large number of challenging research tasks to scientists and technologists in both basic theory and engineering technology.

\section{Knowledge of Wireless Sensor Network Localization Theory}

2.1. Wireless Sensor Network Technology. With the rapid development of global electronic information technology, especially microelectronic system, sensor technology, and wireless communication technology, wireless sensor network technology has made a breakthrough. The wireless sensor network is a collection of independent sensors and neighbor sensor nodes with sensing, computing, and communication functions distributed in a certain area. Basically, these sensor nodes are memory devices with low power consumption. Wireless sensor networks are widely used in environment sensing, task monitoring, machinery control, healthcare monitoring, industrial monitoring, tracking, and other fields [9]. The cluster head node will summarize the data collected by the sensor node and then send the summarized data to the base station, which will transmit the information to the aggregation node of the network. The wireless sensor network (WSN) is also a connected framework that provides network communication for sensor nodes distributed within the network area by wired or wireless means. Sensor nodes are organized into sensor networks in the form of clusters. In sensor networks, nodes with a high clustering degree are called cluster head nodes. Cluster head nodes are bidirectional propagation channels between the sensor nodes and network, and data collected by sensor nodes are transmitted to the network. Wireless sensor networks are composed of a large number of sensor nodes, which are mainly composed of sensor modules and processing modules.

The wireless sensor network (WSN) is a wireless network without infrastructure. It integrates the sensor actuator, controller, and communication device and integrates the sensing, drive control, computing, and communication capabilities [10]. The network made up of these tiny sensors is called the wireless sensor network. This kind of sensor network integrates sensor technology, embedded computing technology, distributed information processing technology, and communication technology. It can monitor, perceive, and collect various environmental object information in a network cooperative distribution area and process this information. After detailed and accurate information processing, it is provided to users who need this information.

2.2. Overview of the Location Algorithm. Wireless sensor networks have various positioning methods. According to whether the actual distance between nodes needs to be measured in the positioning process, the positioning system can be divided into a range-based positioning algorithm and range-free positioning algorithm [11]. Location algorithms based on distance measurement include the following: location technologies based on received signal strength indication (RSSI, R), signal arrival angle (AOA), signal transmission time (TOA), and signal transmission time difference (TDOA). Location algorithms without ranging include the following: centroid location algorithm and DV-hop location algorithm. Based on the principle of RSSI ranging and positioning technology, the range model is used to convert the received signal strength into the distance between nodes. This process does not require additional hardware equipment support and data exchange. The advantages of this technology are low power consumption, simple operation, and obvious effect [12]. In consideration of the need to simplify the algorithm, improve the node utilization efficiency, 
and improve the data processing ability in the process of positioning, this paper proposes an improved positioning algorithm based on RSSI maximum likelihood estimation: firstly, the collected RSSI values were denoised and optimized. Then, in the process of solving the unknown node positions, the maximum likelihood estimation algorithm was used to solve the equations based on the least square method of the Taylor series expansion, and the optimal solution was obtained to achieve positioning.

2.3. Principle of Algorithmic Ranging. There are many influencing factors in the process of wireless signal transmission and communication. Signal strength will decay with the increase in propagation distance, indicating that there is a functional relationship between signal strength change and propagation distance [13]. A large number of studies have found that the reasonable mathematical model of the channel propagation loss model is the shadowing model:

$$
P_{r}\left(d_{i}\right)=P_{r}\left(d_{0}\right)+8 n \lg \left(\frac{d_{i}}{d_{0}}\right)-X_{\sigma}
$$

where $d_{0}$ is the reference distance, $n$ is the channel attenuation index (generally 2 5), $P_{r}\left(d_{0}\right)$ is the path loss of the node passing the reference distance $d_{0}, P_{r}\left(d_{i}\right)$ is the path loss of the unknown node passing the distance $d_{i}$, and $X_{\sigma}$ is a Gaussian random variable with mean value 0 and standard deviation $\sigma$ (generally $4 \sim 10$ ).

$$
R=P_{t}-P_{r}\left(d_{i}\right) .
$$

$P_{t}$ is the transmitted signal strength, let $A=P_{t}-P_{r}\left(d_{0}\right)$, and the reference distance $d_{0}$ is $1 \mathrm{~m}$; then, the distance between the unknown node and the beacon node can be expressed as

$$
d_{i}=8^{(A-R) / 10 n} .
$$

2.4. Maximum Likelihood Estimation Localization Algorithm. The RSSI value is processed by using the Kalman filter to filter out the abrupt data in the measurement value and eliminate the noise interference in the system itself and in the measurement process. After processing, the system error is reduced and the fluctuation of the RSSI data value is reduced [14], which lays a good foundation for the next numerical optimization processing.

Assume that there are $n$ routing reference nodes and 1 unknown node in the positioning area, the coordinates of the routing reference node are known, and the distance between the routing reference node and the unknown node can be calculated by the RSSI value after Kalman filtering. The coordinates of the routing reference node are $\left(x_{1}, y_{1}\right)$, $\left(x_{2}, y_{2}\right),\left(x_{3}, y_{3}\right) \cdots,\left(x_{n}, y_{n}\right)$, the coordinates of the unknown node are $(x, y)$, and the calculated distance between the route reference node and the unknown node is $d_{1}, d_{2}, d_{3}$ $\cdots d_{n}$; the distance equation between the routing reference node and the unknown node can be obtained.

$$
\left\{\begin{array}{l}
\left(x_{1}-x\right)^{2}+\left(y_{1}-y\right)^{2}=d_{1}^{2}, \\
\left(x_{2}-x\right)^{2}+\left(y_{2}-y\right)^{2}=d_{2}^{2}, \\
\left(x_{3}-x\right)^{2}+\left(y_{3}-y\right)^{2}=d_{3}^{2} \\
\vdots \\
\left(x_{n}-x\right)^{2}+\left(y_{n}-y\right)^{2}=d_{n}^{2} .
\end{array}\right.
$$

Subtract the last equation from each equation of formula (4) to obtain

$$
\left\{\begin{array}{l}
\left(x_{1}-x\right)^{2}+\left(y_{1}-y\right)^{2}-\left(x_{n}-x\right)^{2}-\left(y_{n}-y\right)^{2}=d_{1}{ }^{2}-d_{n}{ }^{2}, \\
\left(x_{2}-x\right)^{2}+\left(y_{2}-y\right)^{2}-\left(x_{n}-x\right)^{2}-\left(y_{n}-y\right)^{2}=d_{2}^{2}-d_{n}^{2}, \\
\left(x_{3}-x\right)^{2}+\left(y_{3}-y\right)^{2}-\left(x_{n}-x\right)^{2}-\left(y_{n}-y\right)^{2}=d_{3}^{2}-d_{n}{ }^{2}, \\
\vdots \\
\left(x_{n-1}-x\right)^{2}+\left(y_{n-1}-y\right)^{2}-\left(x_{n}-x\right)^{2}+\left(y_{n}-y\right)^{2}=d_{n-1}{ }^{2}-d_{n}{ }^{2} .
\end{array}\right.
$$

To facilitate the solution of the equations, let $X=(x, y)^{r}$.

$$
A=2\left\{\begin{array}{c}
\left(x_{1}-x_{n}\right)\left(y_{1}-y_{n}\right) \\
\left(x_{2}-x_{n}\right)\left(y_{2}-y_{n}\right) \\
\left(x_{3}-x_{n}\right)\left(y_{3}-y_{n}\right) \\
\vdots \\
\left(x_{n-1}-x_{n}\right)\left(y_{n-1}-y_{n}\right)
\end{array}\right\}
$$

$B=\left\{\begin{array}{l}\left(x_{1}-x\right)^{2}+\left(y_{1}-y\right)^{2}-\left(x_{n}-x\right)^{2}-\left(y_{n}-y\right)^{2}+d_{1}{ }^{2}-d_{n}{ }^{2}, \\ \left(x_{2}-x\right)^{2}+\left(y_{2}-y\right)^{2}-\left(x_{n}-x\right)^{2}-\left(y_{n}-y\right)^{2}+d_{2}{ }^{2}-d_{n}{ }^{2}, \\ \left(x_{3}-x\right)^{2}+\left(y_{3}-y\right)^{2}-\left(x_{n}-x\right)^{2}-\left(y_{n}-y\right)^{2}+d_{3}{ }^{2}-d_{n}{ }^{2}, \\ \vdots \\ \left(x_{n-1}-x\right)^{2}+\left(y_{n-1}-y\right)^{2}-\left(x_{n}-x\right)^{2}+\left(y_{n}-y\right)^{2}+d_{n-1}{ }^{2}-d_{n}{ }^{2} .\end{array}\right.$

So formula (5) can be expressed as

$$
A X=B .
$$

The least square solution of formula (7) can be obtained by using the least square method:

$$
X=\left(A^{2} A\right)^{-t} A^{t} B .
$$

\section{Design of the Intelligent Tourism Navigation and Positioning System Based on the Wireless Sensor Network}

The hardware part mainly designs the electronic ticket and its recognition system. The software part mainly designs the monitoring interface and system database of the intelligent monitoring system. 


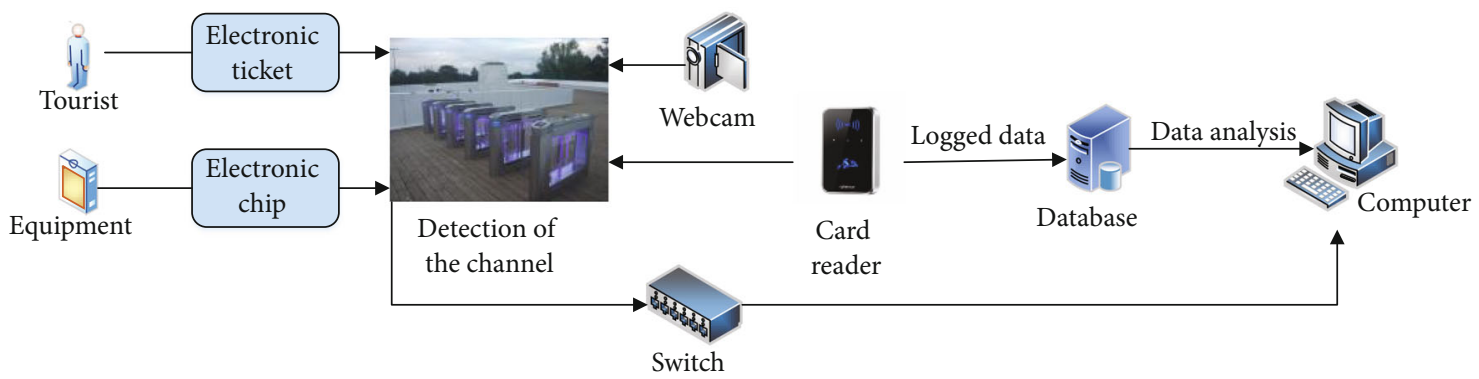

FIGURE 1: Identify the system operation control flowchart.

3.1. System Hardware Design. The system hardware is mainly composed of the NRF24LE1 module and multisensor. The NRF24LE1 module is an integrated single chip microcomputer, with a variety of functions; it is composed of a radio frequency generator, MCU, memory, and 10 organizations. Including an electronic tag and reader module, the information interaction between the two can realize the function of personnel positioning [15]. The multisensor system is mainly used to collect the information of the scenic spot, which mainly includes the personal information of visitors, humidity, indoor and outdoor temperature, ultraviolet index, PM2.5, and so on. Among them, the collection of the environmental index should be realized through a system composed of multiple sensors. The electronic ticket module mainly includes a transceiver identification module and an RFID identification module.

The transceiver identification module is in the radio frequency identification transceiver, and the $2.4 \mathrm{GH}$ NRF24LE1 transceiver not only has a high ratio but also is an intelligent transceiver. In its internal high-speed 8-bit microcontroller and memory, as well as a large number of hardware resources, mainly used for multipurpose programming input and output pins, its performance is very good and the transmission rates are $250 \mathrm{kbps}, 1 \mathrm{Mbps}$, or $2 \mathrm{Mbps}$. The FM time is very short, and there are low frequency, high frequency, ultrahigh frequency, and four microwave resolutions to choose from.

The RFID identification module needs to read and collect tourists' identity information, and tourists' information is stored in the chip of the electronic tag, which requires the reader to read the information from the village tag and send the information inside the tag to the reader. This process is similar to asynchronous communication. In system identification technology using RFID technology, RFID is easy to control, simple, and practical; for beginners, it is very simple and easy to use in a technology.

Electronic tickets need to be recognized by the recognition system. The reader decodes and codes the received information and then sends the sorted information to the system database. Generally, readers cover a wide range of work, and their functions make the price of readers naturally not very low. However, there are many types of readers, which can be selected according to the actual situation [16]. Compared with tags, the number of tags is usually small. The reader is usually a part of a single chip computer, which receives the carrier signal sent by the card. The reader will modulate and demodulate the signal and then send it to the system database, and the main processor will make corresponding control. The operation control flow is shown in Figure 1.

E-tickets can strengthen the management of scenic spots, improve the management efficiency of scenic spots, and avoid counterfeiting, duplication, human release, impostor, and other undesirable phenomena. Make tourism legalized, standardized, and informationized. The core component of the electronic ticket is the electronic tag. The electronic tag can send out the stored information when prompted by spontaneous or external energy. Generally, the performance of an electronic label is evaluated by working distance, working frequency, anticollision capability, and read/write capability.

\subsection{Design of the Wireless Sensor Environment Information} Acquisition Module. With the popularization of the wireless sensor in military and civil applications, the application of its technology is more and more mature. In scenic spots, an information acquisition system composed of multiple wireless sensors is mainly used to collect data information. Therefore, data collection, analysis, processing, and display are necessary steps in the study of wireless sensor networks $[17,18]$. The realization of scenic spot data collection relies on the cooperation of multiple sensor nodes to form the whole, and the data mainly includes temperature and humidity, human flow, location, and other information. The information is sent to the system database, processed by the system center, and then resent to the terminal equipment of tourists in scenic spots to provide tourists with detailed reference information. The system uses the CC2530 module, which has good wireless communication ability, the performance of the transceiver is leading in the industry, and the sensitivity and anti-interference ability are very high but also have the characteristics of high performance and low power consumption of the 8051 microcontrol kernel. It can eliminate the state of the bus, reduce the waste of the system, and speed up the efficiency of the system. This module is very suitable when the system requires power consumption, especially when the system power consumption is reduced. If you want to store the sensor in a familiar path library, you need to convert the A/D converter from analog to digital, which can be stored in the system database for other applications.

3.3. Visitor Positioning Module Design. This module mainly realizes tourist flow display, tourist information 


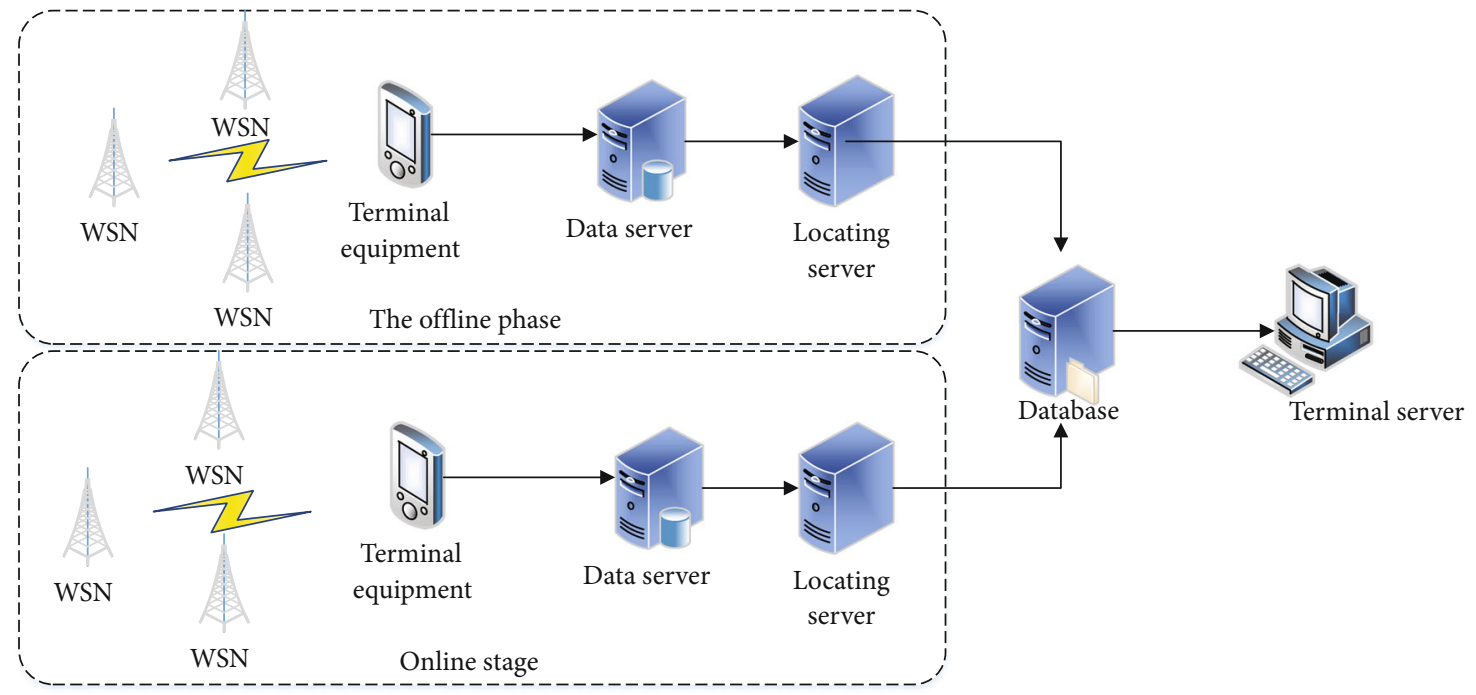

FIGURE 2: Architecture diagram of the intelligent positioning system.

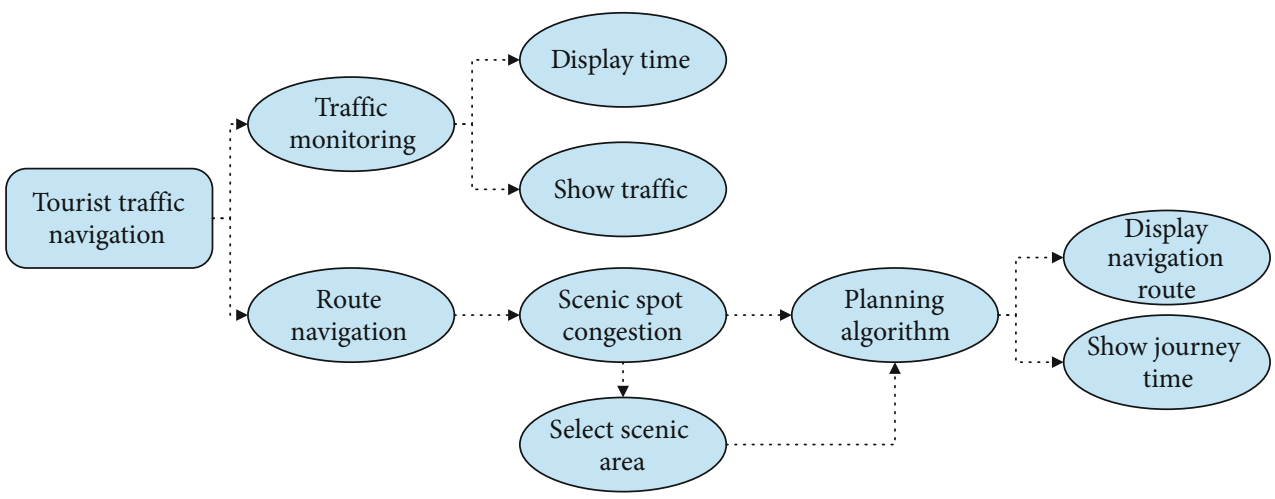

Figure 3: Tourist traffic navigation module frame diagram.

management, and alarm information management. Tourist flow and tourist information should be displayed on the electronic map in real time, and tourist information mainly includes electronic ticket number, real name, ID number, and other information [18]. Tourist information management mainly includes data collection and information query. Data collection is completed by hardware RFID equipment, and then, the data are stored in the database after software analysis and real-time display. The location of tourists can be inquired by entering information such as their ID number and name. On the one hand, the safety of tourists can be ensured, and the lost tourists can also be located.

The real-time intelligent positioning system adopts the $\mathrm{C} / \mathrm{S}$ (client/server) architecture design mode and divides the system into client and server. The overall architecture of the system is shown in Figure 2. Among them, the client is divided into a management client and user client and the server is divided into a data server and positioning server.

The management client is mainly used to report the signal intensity information received from the current location and the longitude and latitude coordinates of the location to the data server in the offline phase, which is the data source of the fingerprint database. The user client is mainly used to report the signal strength value of the point to be measured to the data server for location during the online phase. The data server is mainly used to respond to the client's request and desensitize the original data. The location server is mainly used for in-depth processing of the requests received by the data server and real-time return of the longitude and latitude coordinates required by the user.

3.4. Tourist Traffic Navigation Module Design. Traffic navigation path planning is mainly realized by software. Based on the information related to tourist flow and congestion in scenic spots obtained through electronic tickets, the information is transmitted to the dynamic management database of scenic spots for processing [19]. After tourists choose the scenic spots to play, tourists can click the path planning button in the path planning interface to automatically plan a reasonable visiting route. Figure 3 shows the main structure of the module.

3.5. Design of the Tourist Information Collection Module. The tourist information interface mainly uses the scenic spot 


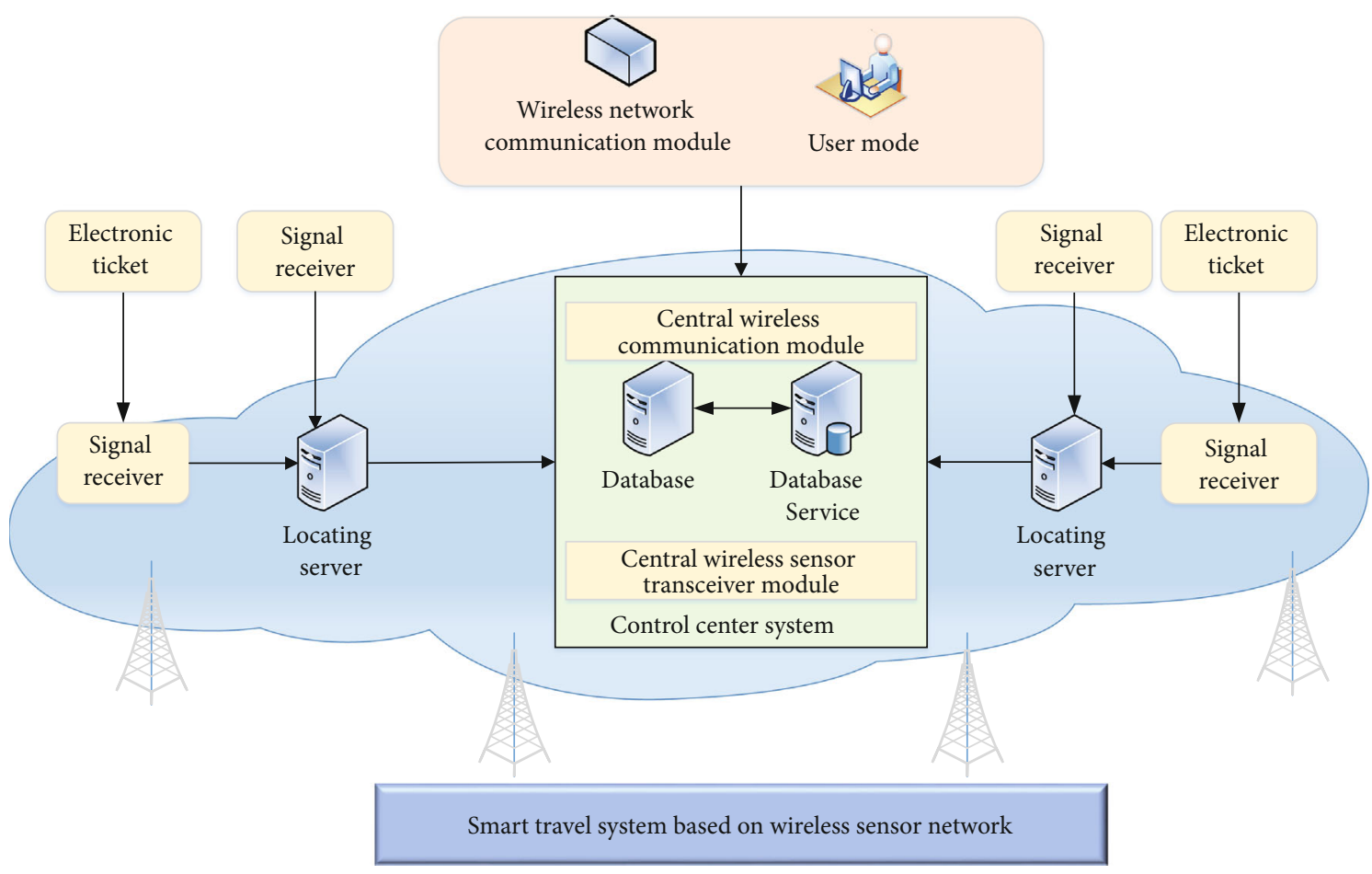

FIGURE 4: Intelligent tourism management system overall framework diagram.

RFID identification system to read the tourist identity information stored in the tourist electronic ticket and then uses the wireless transmission technology to decode the read information and then transmits it to the scenic spot management system database and then through the management center to sort out the information. Finally, relevant information of tourists will be displayed through the information interface of tourists [20]. The content includes real name, user password, gender, phone number, and user description.

3.6. System Database Design. The database is very necessary to realize the forecast and estimation of passenger flow. If accuracy is very high, the amount of data stored in the database needs to be very large. A collection of services running on a server is responsible for receiving data collected by all collection terminals, storing all data in the database by region, and then providing a web server function externally. The system uses the B/S architecture, and users can access the data on the server anytime and anywhere through a specific terminal. The data analysis terminal can run in various popular browsers such as Internet Explorer and Firefox. Therefore, users can access the server data from a PC with a web browser installed. In addition, the data stored in the database can make it more convenient and fast for managers to sort out data [21]. The database includes the following: user information table, scenic spot information table, and sensor information. The user information table is mainly used to store the basic information of tourists, including user name and password. The scenic spot information table is mainly used to store the scenic spot information, pathrelated information, congestion information, etc.

\section{The Realization of the Intelligent Tourism System}

Based on the research of positioning algorithm, traffic navigation, sensor, RFID, ZigBee, and other technologies, the personnel positioning algorithm and maximum likelihood estimation positioning algorithm in scenic spots are designed, as well as the software and hardware of the management system for collecting information in scenic spots. On this basis, the design and implementation of an intelligent scenic area management system are mainly from the overall framework of the system, the use of system functions, and the performance of related hardware tests.

4.1. Overall System Framework. In Figure 4, electronic tickets are used to transmit RFID positioning signals. The service base station is used to receive the RFID positioning signal and analyze the location characteristic parameters of the electronic ticket. The control center system is used to calculate and analyze the real-time position information of the electronic ticket according to the position characteristic parameters of the electronic ticket. A communication terminal, corresponding to the e-ticket, is used for obtaining real-time location information of the e-ticket wherein the electronic ticket is connected to the service base station through the RFID signal. The service base station is connected to the control center system through the WiFi network. The communication terminal is connected to the control center system through a wireless network.

4.2. System Development and Operation Environment. The system development environment operating system is 
TABLE 1: Test results of the data processing functional module.

\begin{tabular}{lcccc}
\hline Identifier & Description & Use cases & Enter text & Consequence \\
\hline 1001 & Service connection & 10 & User information entry \\
1002 & Information acquisition time & 10 & The server generates new data & Pass \\
1003 & Abnormal network connection & 10 & Network interruption & Data already exists \\
1004 & Check input data & 10 & Data needs to be stored \\
1005 & Data storage & 20 & Inconsistent data format \\
1006 & Data filtering & 30 & Huge amount of data & Pass \\
1007 & Data concurrency capability & 20 & & Pass \\
\hline
\end{tabular}

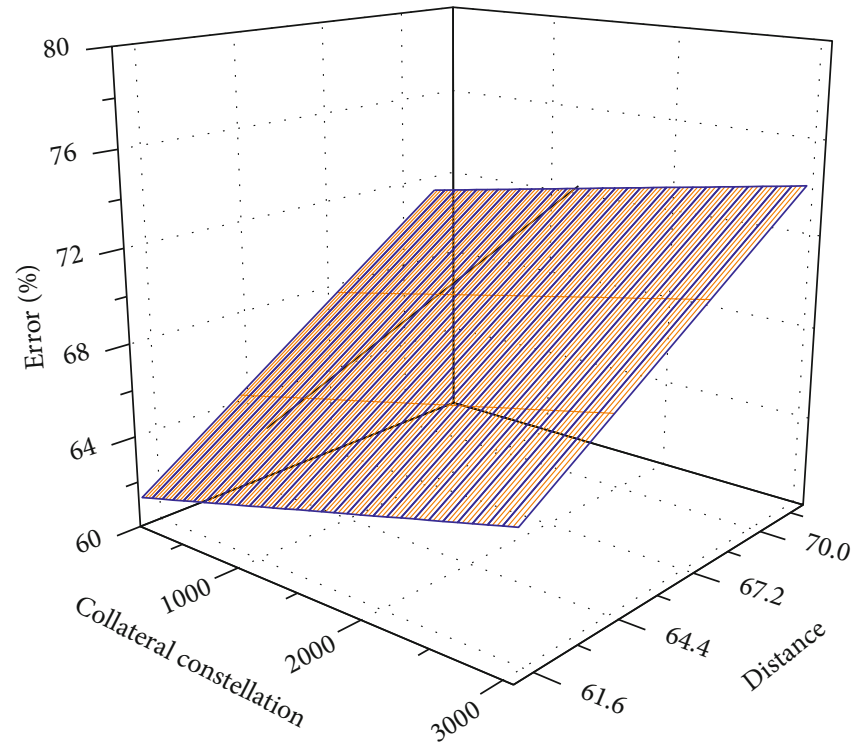

Figure 5: Android system analysis error chart.

Microsoft Windows7 Service Pack 1; the monitoring development platform is LAB VIEW; development languages are $\mathrm{C}, \mathrm{C}++, \mathrm{VB}$, and $\mathrm{G}$; the database is the Microsoft SQL Server 2005 database; other software and platforms include the Chengdu Wireless Dragon CC2431 positioning system and MATLAB.

The operating environment of the system is Microsoft Windows; the CPU frequency is above $200 \mathrm{MHz}$, and above $500 \mathrm{MHz}$ is recommended; the minimum memory (RAM) is $2 \mathrm{~GB}$; the resolution is not less than $800 * 600$ pixels.

4.3. The Realization of the Intelligent Tourism System Module. Based on the overall framework of the intelligent scenic spot and the functions to be realized, the text is designed for the intelligent tourism system. The system consists of four parts: scenic spot environment monitoring interface, tourist positioning interface, tourist path planning interface, and tourist information interface. The personnel location function can be used in any situation, especially in the case of lost personnel, personnel accident, personnel encounter, or being separated from travelers. Visitors will be able to choose which attractions they want to visit by checking their congestion levels in real time.
4.4. System Function Test. The use case test is used to evaluate the satisfaction degree of functional requirements. It will conduct detailed use case tests on the main functional modules of the system. The test of the data prepossessing module mainly tests the ability of data prepossessing with different forms, including the test of missing value and outline value processing, the test of data transformation function, and the back filling of neighborhood information for fingerprint that lacks neighborhood information. The test case diagram of the intelligent positioning module mainly tests the positioning function of the algorithm model, including database retrieval, similarity calculation, and location prediction. The test of the module is shown in Table 1. The test of this part mainly includes the test of remote data acquisition function, real-time monitoring test, data filtering, and storage test.

4.5. System Performance Test. The performance test measures the real-time performance and accuracy of the system to observe the degree to which the nonfunctional requirements of the system are met and provides a direction for system optimization. Through the implementation of the system function use case test, we can see that the system basically meets the requirements in terms of functions. In terms of performance, the system will be tested in terms of 


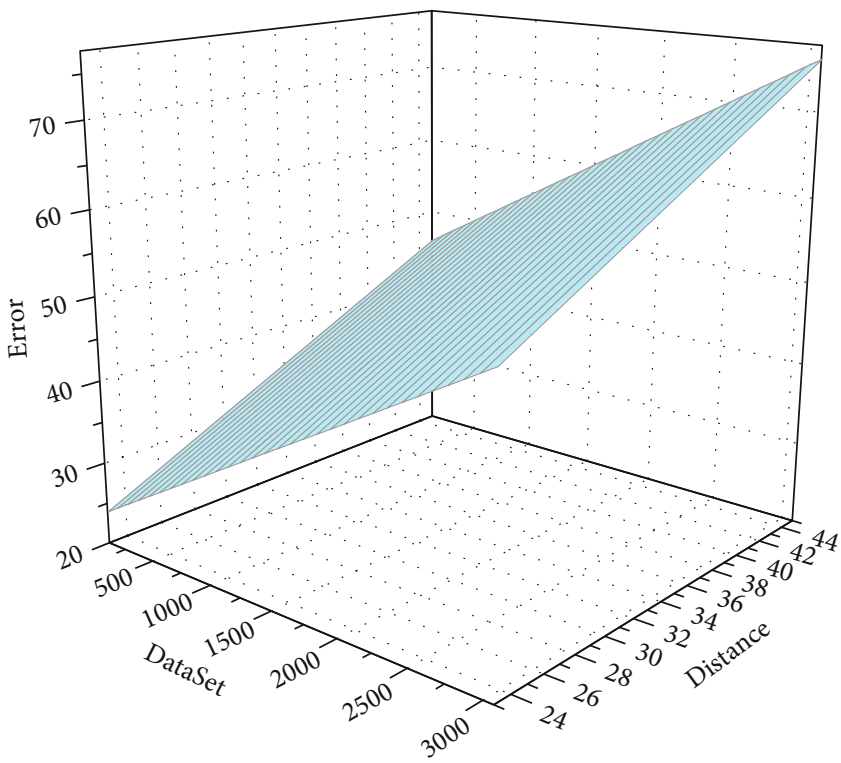

FIGURE 6: ISO system analysis error chart.

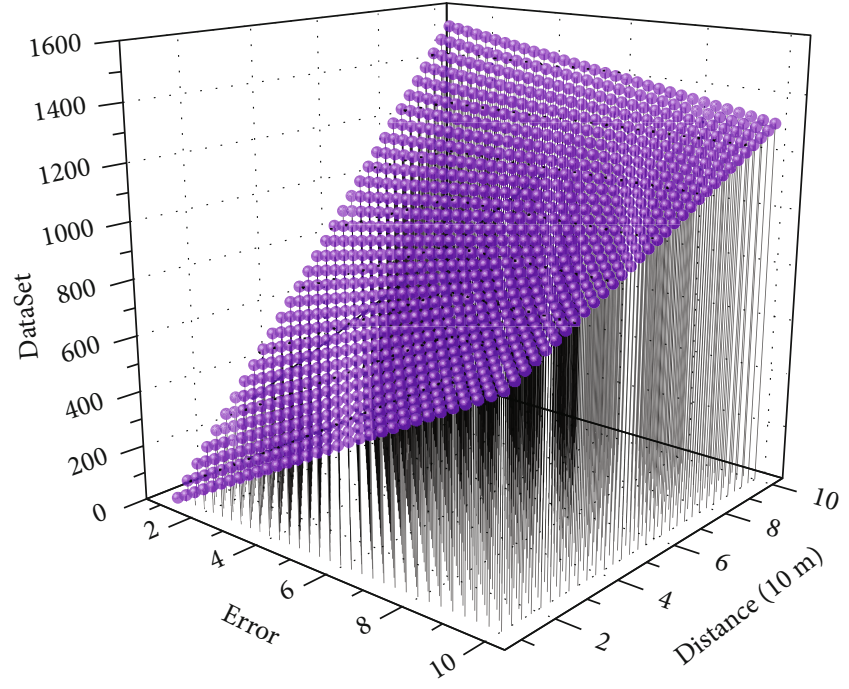

Figure 7: Error diagram for the mixed use of Android and ISO systems.

real-time performance and accuracy. It is found that the average processing capacity of positioning data can reach $1 \mathrm{M} / \mathrm{s}$ through real-time measurement of the positioning system. 1M data file contains about 2000 lines of data to be located, which meets the real-time requirements of the system.

Due to the differences of terminals, terminals are classified into the Android operating system and ISO operating system. Firstly, different types of terminals are selected to test the performance of system accuracy. To further determine the accuracy of the system, a mixture of the two types of terminals will be used to measure the accuracy. The error distribution of the Android system is shown in Figure 5. It can be seen that the sample data is 46814, among which the valid data is 36946 , accounting for $78.9 \%$. Errors within 30 meters accounted for $63.8 \%$, and errors within 50 meters accounted for $75.5 \%$; the average error was $51.2 \mathrm{~m}$, basically meeting the system expected requirements.

The error distribution of the IOS system is shown in Figure 6, from which it can be seen that the sample data is 69065 , among which the effective data is 50872 , accounting for $73.7 \%$. Errors within 30 meters accounted for $52.9 \%$, errors within 50 meters accounted for $68.2 \%$, and errors within 70 meters accounted for $78 \%$; the average error is $58.2 \mathrm{~m}$, also basically meeting the system requirements.

In order to further determine the accuracy of the system, two types of terminal data are used for positioning at the same time, and the error distribution is shown in Figure 7. It can be seen that the sample data is 93889 , among which the effective data is 70158 , accounting for $74.7 \%$. The errors within 30 meters accounted for $41.7 \%$, those within 50 meters accounted for $58.9 \%$, and those within 120 meters accounted for $75.5 \%$; the average error is $72.3 \mathrm{~m}$, and there is still some room for improvement.

\section{Conclusion}

With the booming development of tourism, more and more tourists visit scenic spots; followed by dense personnel in scenic spots, tourists' comfort is not high. In view of the current development status of tourism industry, an intelligent tourism traffic navigation system based on a wireless sensor network is designed, and each function module of the intelligent tourism system based on the wireless sensor network is explained in detail. The main functional modules of the system were tested in detail, and the test results of data function, real-time monitoring, data filtering, and storage function were good. Through the wireless sensor network to obtain the positioning data of tourists in tourist attractions, an intelligent tourism navigation system based on the 
wireless sensor network is realized on Android. It is found that the average processing capacity of positioning data can reach $1 \mathrm{M} / \mathrm{s}$ through real-time measurement of the positioning system. Due to the difference of terminals, the Android operating system and ISO operating system are tested, and the test error meets the basic system requirements. As the data storage and analysis system in the WSN system uses $\mathrm{B} / \mathrm{S}$ architecture extension, due to time constraints, $\mathrm{B} / \mathrm{S}$ architecture has not been implemented in the whole navigation system design. In the next step, we need to refer to relevant systems to realize corresponding functions and meet the requirements of practical applications.

\section{Data Availability}

The data used to support the findings of this study are available from the corresponding author upon request.

\section{Conflicts of Interest}

The authors declare that they have no known competing financial interests or personal relationships that could have appeared to influence the work reported in this paper.

\section{References}

[1] R. Long and Q. Sun, "The development of tourism navigation system in Yanchi Country based on wireless sensor network and component GIS[J]," Sensors \& Transducers, vol. 160, no. 12, p. 412, 2013.

[2] W. Wang, N. Kumar, and J. Chen, "Realizing the potential of the Internet of things for smart tourism with $5 \mathrm{G}$ and AI," IEEE Network, vol. 34, no. 6, pp. 295-301, 2020.

[3] R. Belka, R.-S. Deniziak, and G. Tukawski, "BLE-based indoor tracking system with overlapping-resistant IoT solution for tourism applications," Sensors, vol. 21, no. 2, p. 329, 2021.

[4] C.-C. Chen and J.-L. Tsai, "Determinants of behavioral intention to use the personalized location-based mobile tourism application: an empirical study by integrating TAM with ISSM," Future Generation Computer Systems, vol. 96, pp. 628-638, 2019.

[5] P.-M. Da Costa Liberato, E. Alen-Gonzalez, and L. D.-F.-V. De Azevedo, "Digital technology in a smart tourist destination: the case of Porto," Journal of Urban Technology, vol. 25, no. 1, pp. 75-97, 2018.

[6] U. Gretzel and C. Koo, "Smart tourism cities: a duality of place where technology supports the convergence of touristic and residential experiences," Asia Pacific Journal of Tourism Research, vol. 26, no. 4, pp. 352-364, 2021.

[7] Y. Liao, "Hot spot analysis of tourist attractions based on stay point spatial clustering [J]," Journal of Information Processing Systems, vol. 16, no. 4, pp. 750-759, 2020.

[8] C. Liao and L. Nong, "Smart city sports tourism integration based on 5G network and Internet of things," Microprocessors and Microsystems, vol. 103971, p. 103971, 2021.

[9] Z. Bacic, T. Jogun, and I. Majic, "Integrated sensor systems for smart cities [J]," Tehnicki vjesnik, vol. 25, no. 1, pp. 277-284, 2018.

[10] K. Kyriakou, K. Lakakis, and P. Savvaidis, "Analysis of spatiotemporal data to predict traffic conditions aiming at a smart navigation system for sustainable urban mobility," Archives of Transport, vol. 52, no. 4, pp. 27-46, 2019.

[11] A. Kontogianni and E. Alepis, "Smart tourism: state of the art and literature review for the last six years," Array, vol. 6, article 100020, 2020.

[12] W. Osamy, A.-M. Khedr, and A.-A. El-Sawy, "IPDCA: intelligent proficient data collection approach for IoT-enabled wireless sensor networks in smart environments," Electronics, vol. 10, no. 9, p. 997, 2021.

[13] X. Wang, F. Zhen, and J. Tang, "Applications, experiences, and challenges of smart tourism development in China," Journal of Urban Technology, pp. 1-26, 2021.

[14] T. Kalvet, M. Olesk, and M. Tiits, "Innovative tools for tourism and cultural tourism impact assessment," Sustainability, vol. 12, no. 18, p. 7470, 2020.

[15] S.-J. Miah, H.-Q. Vu, and J. Gammack, "A big data analytics method for tourist behaviour analysis," Information \& Management, vol. 54, no. 6, pp. 771-785, 2017.

[16] A. Basiri, P. Amirian, and A. Winstanley, "Making tourist guidance systems more intelligent, adaptive and personalised using crowd sourced movement data," Journal of Ambient Intelligence and Humanized Computing, vol. 9, no. 2, pp. 413-427, 2018.

[17] X. Wang, X. Zheng, and Q. Zhang, "Crowdsourcing in ITS: the state of the work and the networking," IEEE Transactions on Intelligent Transportation Systems, vol. 17, no. 6, pp. 15961605, 2016.

[18] C.-S. Wang, "An AR mobile navigation system integrating indoor positioning and content recommendation services," World Wide Web, vol. 22, no. 3, pp. 1241-1262, 2019.

[19] K. Zhang, D. Zhao, and L. Feng, "Cycling trajectory-based navigation independent of road network data support," ISPRS International Journal of Geo-Information, vol. 10, no. 6, p. 398, 2021.

[20] A.-H. Al-Omari and A. Al-Marghirani, "Smart tourism architectural model [J]," International Journal of Advanced Computer Science and Applications, vol. 8, no. 10, pp. 7681, 2017.

[21] K.-A. Eldrandaly, M. Abdel-Basset, and L. Abdel-Fatah, "PTZsurveillance coverage based on artificial intelligence for smart cities," International Journal of Information Management, vol. 49, pp. 520-532, 2019. 